\title{
Exact solution of Ising model on a small-world network
}

\author{
J. Viana Lopes, Yu. G. Pogorelov, and J. M. B. Lopes dos Santos \\ Centro de Física do Porto, Departamento de Física, Faculdade de Ciências, Universidade do Porto, $4169-007$ Porto, Portugal \\ R. Toral \\ Instituto Mediterraneo de Estudios Avanzados IMEDEA (CSIC-UIB), Ed. Mateu Orfila, Campus E-07122 Palma de Mallorca, Spain
}

(Received 14 February 2004; published 25 August 2004)

\begin{abstract}
We present an exact solution of a one-dimensional Ising chain with both nearest-neighbor and random long-range interactions. Not surprisingly, the solution confirms the mean-field character of the transition. This solution also predicts the finite-size scaling that we observe in numerical simulations.
\end{abstract}

DOI: 10.1103/PhysRevE.70.026112

PACS number(s): 05.50.+q, 64.60.Cn, 05.70.Jk

\section{INTRODUCTION}

Physical interactions are usually of limited range. Nevertheless, there is a long history of study of the effect of infinite range interactions in basic models of statistical physics $[1,2]$. More recently, the work of Watts and Strogatz [3] on small world networks brought renewed attention to this question. Watts and Strogatz considered a quantitative model that interpolates between a regular lattice and a random one. They showed that the addition of a small fraction of random long range links can dramatically change the connectivity properties of the lattice. In particular, in a lattice of $N$ nodes the averaged chemical distance, that is the averaged minimum number of links between any two nodes, is of order $\mathcal{O}(\ln N)$, rather than $\mathcal{O}(N)$, as in a regular lattice, regardless of the number $p N$ of additional links $(p>0)$, provided $N$ is large enough. Clearly, this change should be reflected in the phenomenology of any physical models defined on such lattices. Researchers were quick to grasp this opportunity and many physical models and processes have been considered in small-world networks, like cellular automata [4], diffusion [5], neural networks [6], the spread of disease [7], and many others [8].

The Ising model, the simplest paradigm of order-disorder transitions, has been studied on small-world networks in one dimension (1D) both numerically and analytically [9-12], and in two (2D) and three dimensions (3D) numerically [13]. Some partial analytic results, based on the use of the replica trick, were presented by Gitterman [11] and Barratt and Weigt [10]. They are, however, contradictory (Gitterman predicts a ferromagnetic phase only for $p \geqslant 1 / 2$ ) and, at any rate, these results do not constitute a full solution of the thermodynamics of the model. There is a consensus that the ferromagnetic Ising model has a mean-field transition for any finite value of $p$ even in 1D, even though Hastings predicts the occurrence of anomalous scaling of the mean-field amplitudes with $p \rightarrow 0$ [14]. Dorogovtsev et al. [15] presented a very general discussion, for networks that are locally treelike, based on the solution of the Ising model on a Bethe lattice [16].

In this work, we present an explicit solution of the 1D Ising model with additional random long-range bonds. We are able to calculate not only the transition temperature, but also the complete thermodynamics at all temperatures as a function of $p$ and of the strength of long range interactions $I$ (though in this paper, we only present the results for the free energy and specific heat, in zero field). The form of finite size scaling corrections to the thermodynamics can also be derived from our solution.

The following Sec. II presents a description of our specific model and the basic algorithm for its exact solution. The combinatorial treatment of its constituents is given is Sec. III. In Sec. IV, we analyze the resulting thermodynamics and conclude on the mean-field character of the ordering transition. Section V shows how the finite size scaling properties can be obtained within the same approach. Finally, the relation between our results and those of Dorogovtsev et al. is discussed in Sec. VI.

\section{MODEL}

Watts and Strogatz originally considered a model in which the bonds of a regular lattice are rewired at random with a probability $p$. It is widely believed that the modified model, in which random long-range bonds, or shortcuts, are added to the regular lattice [7], is essentially equivalent.

The actual model consists of a chain of $N$ Ising spins $\sigma_{i}$, with nearest-neighbor interactions $J$ (chain bonds) and shortcut interactions $I$ (long range bonds), both $J$ and $I$ being positive, so that the Hamiltonian reads

$$
\mathcal{H}=-J \sum_{i=0}^{N-1} \sigma_{i} \sigma_{i+1}-I \sum_{(i j) \in S} \sigma_{i} \sigma_{j}-h \sum_{i=0}^{N-1} \sigma_{i},
$$

with $\sigma_{i}= \pm 1$ and periodic boundary conditions: $\sigma_{0}=\sigma_{N}$. The set $S$ contains $N_{b}=p N$ shortcut pairs of spins, and the last term accounts for the effect of external magnetic field. We present in detail the solution of a version of this model in which the sites connected by shortcuts are equally spaced in the regular lattice, a distance $1 / 2 p$ apart, but the shortcuts are randomly arranged among these spins. The solution of the original model, in which the $2 p N$ sites are randomly distributed along the lattice, turns out to be essentially the same, and some results for this latter case are also presented in the Appendix. In either case, the bond selection is such that no spin is linked to more than one shortcut. 
We consider a transformation from site variables to bond variables, which we formulate for a general Ising model in zero-magnetic field,

$$
\mathcal{H}=-\sum_{(i, j)} J_{i j} \sigma_{i} \sigma_{j},
$$

where $J_{i j}$ can be chosen arbitrary (though in what follows they are only $0, J$, or $I$ ). The partition function for temperature $T=\beta^{-1}$ is given by

$$
\mathcal{Z}=\operatorname{Tr}_{\{\sigma\}} \exp \left(\beta \sum_{(i, j)} J_{i j} \sigma_{i} \sigma_{j}\right)=\operatorname{Tr}_{\{\sigma\}} \prod_{(i, j)} \exp \left(\beta J_{i j} \sigma_{i} \sigma_{j}\right) .
$$

We can use the known identity

$$
\exp \left(\beta J_{i j} \sigma_{i} \sigma_{j}\right)=\cosh \beta J_{i j}\left(1+\sigma_{i} \sigma_{j} \tanh \beta J_{i j}\right),
$$

and then represent the latter factor as a sum in a discrete "bond variable" $b_{i j}=0,1$ :

$$
1+\sigma_{i} \sigma_{j} \tanh \beta J_{i j}=\sum_{b_{i j}=0,1}\left(\sigma_{i} \sigma_{j} \tanh \beta J_{i j}\right)^{b_{i j}},
$$

to write the partition function as

$$
\begin{aligned}
\mathcal{Z}= & \left(\prod_{(i, j)} \cosh \beta J_{i j}\right) \\
& \times \operatorname{Tr}_{\{\sigma\}} \sum_{\{b\}} \prod_{(i, j)}\left(\sigma_{i} \sigma_{j} \tanh \beta J_{i j}\right)^{b_{i j}} .
\end{aligned}
$$

When we trace over any spin variable $\sigma_{i}$, in a product with fixed configuration $\{b\}$ of bond variables $b_{i j}$, we get zero if the $i$ th spin multiplicity in this product, $\Sigma_{j} b_{i j}$, is odd, and a factor of 2 if it is even. Therefore, we can trace over the spin variables to obtain

$$
\begin{aligned}
\mathcal{Z}= & 2^{N}\left(\prod_{(i, j)} \cosh \beta J_{i j}\right) \\
& \times \sum_{\{b\}} \prod_{(i, j)}\left(\tanh \beta J_{i j}\right)^{b_{i j},},
\end{aligned}
$$

where the sum over $\{b\}$ is restricted to configurations with only even $\Sigma_{j} b_{i j}$ for all $i$.

As an example, we can derive from Eq. (9) the partition function of the Ising chain $\left(J_{i j}=J\right.$, for nearest neighbors). Since each spin there has only two bonds, this implies that either $b_{i j}=0$ for all the bonds or $b_{i j}=1$ for all the bonds, and these two alternatives contribute in the partition function

$$
\mathcal{Z}_{\text {chain }}=(2 \cosh \beta J)^{N}\left(1+\tanh ^{N} \beta J\right)
$$

as expected.

The restriction of the sum in Eq. (9) to configurations such that $\Sigma_{j} b_{i j}$ is even, allows a reduction of the number of required variables. If the site $i$ has no shortcut bonds, then this condition reduces to $b_{i-1, i}+b_{i, i+1}$ being an even number, that is, $b_{i-1, i}=b_{i, i+1}\left(\right.$ since $\left.b_{i j}=0,1\right)$. On the other hand, if $i$ is a shortcut site, with an associated additional bond variable, the sum in Eq. (9) gets only nonvanishing contributions when $b_{i-1, i}=b_{i, i+1}$, if the additional bond variable is 0 , or when $b_{i-1, i}=1-b_{i, i+1}$, if the additional bond variable is 1 . In any case, the knowledge of one chain bond variable (say $\left.b_{0,1}\right)$ and the shortcut variables is enough to determine all the terms that give a nonzero contribution to Eq. (9). Hence, the partition function can be written as an unrestricted sum over $b_{0,1}$ and $N_{b}$ shortcut bond variables which, in order to simplify notation, we denote simply by $b_{0}, b_{1}, \ldots, b_{N_{b}}$ :

$$
\mathcal{Z}=\mathcal{Z}_{\text {chain }} c_{I}^{p N} \sum_{\left\{b_{0}, \ldots, b_{N_{b}}\right\}} t_{J}^{L[b]} t_{I}^{M[b]},
$$

where $c_{I} \equiv \cosh \beta I, t_{J} \equiv \tanh \beta J, t_{I} \equiv \tanh \beta I, M=\sum_{i=1}^{n} b_{i}$ is the number of shortcut bonds with $b=1$, and $L$ the number of chain bonds with $b=1$. We calculate it as follows: Given a configuration of $N_{b}$ shortcuts and a choice of $M$ from them with $b_{i}=1$, the chain gets divided into $2 M$ segments between consecutive spins connected to one of $M$ bonds. These segments have lengths $l_{1}, \ldots, l_{2 M}$. When $b_{0}=0$, the value $L[b]$ is the sum of even lengths, $l_{2}+l_{4}+\cdots+l_{2 M}$. Otherwise, it is the sum of the odd ones, $l_{1}+l_{3}+\cdots+l_{2 M-1}$.

Our solution is based on the observation that Eq. (11) permits explicit separation of two extensive thermodynamical variables $L[b]$ and $M[b]$ [both being $\mathcal{O}(N)$ ] from $N_{b}$ microscopic variables $b_{i}$. The latter only define a temperature independent prefactor $\Omega(M, L)$ at the product $t_{J}^{L} t_{I}^{M}$ with given $L$ and $M$. A similar situation (but with a single variable $t_{J}^{L}$ ) was already addressed by Saul and Kardar for the problem of Ising spin glasses [17]. Instead of the numerical procedure for the prefactor employed in Ref. [17], we are able here to calculate analytically the sum over all the bond configurations in Eq. (11). Denoting $\Omega(M, L)=\exp [S(M, L)]$ the number of choices of $M$ segments giving a fixed value of $L$, we get

$$
\mathcal{Z}=\mathcal{Z}_{\text {chain }} c_{I}^{p N} \sum_{b_{0}} \sum_{M, L} \exp \left(S-L / \xi_{J}-M / \xi_{I}\right),
$$

where $\xi_{J}=1 / \ln \left(1 / t_{J}\right)$ is the Ising chain correlation radius and $\xi_{I}=1 / \ln \left(1 / t_{I}\right)$.

The crucial property of the exponential function under sum in Eq. (12) is that it has, as will be seen below, an extremely sharp maximum at some macroscopically great values of $L$ and $M$. This reduces the calculation of its contribution to the free energy, $\mathcal{F}=-T \ln \mathcal{Z}$, to the logarithm of its maximum. The latter turns out to be $\mathcal{O}(N)$ and thus a self-averaging quantity, that is coinciding, for (almost) any random realization of the disordered system, with its average value [18].

Passing to the intensive thermodynamical variables $l$ $\equiv L / N, \quad n \equiv M / N_{b}$, and to the intensive function $s(l, n)$ $=S(L, M) / N$, we rewrite Eq. (12) as

$$
\mathcal{Z}=2 \mathcal{Z}_{\text {chain }} c_{I}^{p N} \sum_{l, n} \exp \left[N\left(s-l / \xi_{J}-p n / \xi_{I}\right)\right]
$$

and in the thermodynamic limit $N \rightarrow \infty$, the sum converts into integral which can be done by steepest descent. The values $b_{0}=0$ and 1 give identical contributions, hence the factor 2 . The free energy per spin is $f=\mathcal{F} / N=-(T / N) \ln \mathcal{Z}$ $=-(T / N) \ln \mathcal{Z}_{0} \mathcal{Z}_{a}=f_{0}+f_{a}$, where 


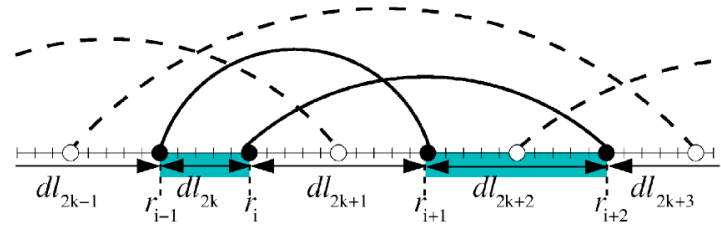

FIG. 1. $\Omega(L, M)$ is the number of possible choices of $M$ bonds, terminating in filled dots, from $p N$ fixed bonds, which have a given value of the sum $l_{2}+l_{4}+\cdots+l_{2 M}=L / d$.

$$
f_{0}=-T[\ln (2 \cosh \beta J)+p \ln \cosh \beta I]
$$

and the "anomalous" term $f_{a}$ (if exists, see below) is given by simple minimization with respect to $l$ and $n$ of the following function:

$$
f(l, n)=T\left[-s(l, n)+l / \xi_{J}+p n / \xi_{I}\right] .
$$

The task that remains is to calculate the configurational entropy $s(l, n) \equiv \ln \Omega(L, M) / N$ (for $L, M=\mathcal{O}(N)$ ).

\section{COMBINATORICS OF BONDS}

The calculation of $\Omega(L, M)$ can be formulated in the following way.

We are given a chain of $N$ sites $\{0,1,2, \ldots, N-1\}$ with periodic boundary conditions. The shortcut sites are evenly spaced forming a regular lattice with coordinates $\{d, 2 d, \ldots, N\}$, where $d=1 / 2 p$. A number $p N$ of shortcuts connect $p N$ pairs, randomly chosen from these sites. If we choose $M$ of these bonds (those for which $b=1$ ) from the total of $p N$, the corresponding shortcut sites (the filled dots in Fig. 1) will have coordinates, in increasing order $\left\{r_{1}, r_{2}, \ldots, r_{2 M}\right\} \subseteq\{d, 2 d, \ldots, N\}$. These site coordinates will divide the lattice into $2 M$ segments of lengths $d l_{1}, \ldots, d l_{2 M}$ where

$$
\begin{gathered}
l_{i}=\frac{r_{i}-r_{i-1}}{d}, \quad i \neq 1, \\
l_{1}=\frac{N-r_{2 M}+r_{1}}{d},
\end{gathered}
$$

and $\sum_{i=1}^{2 M} l_{i}=N / d$. Then $\Omega(L, M)$ is the number of possible choices of the $M$ bonds such that

$$
l_{2}+l_{4}+l_{6}+\cdots+l_{2 M}=L / d .
$$

We are asking in how many ways one can divide the interval $[0, N / d]$ into $2 M+1$ integer lengths, $\left\{l_{1}, \ldots, l_{2 M+1}\right\}$, where only $l_{2 M+1}$ may be zero, so that even lengths are summed to

$$
l_{2}+l_{4}+\cdots+l_{2 M}=L / d .
$$

Then, clearly, the odd lengths should sum to

$$
l_{1}+l_{3}+\cdots+l_{2 M+1}=\frac{N-L}{d} .
$$

Therefore, $\Omega(L, M)$ is just the number of ways of dividing the interval $[0, L / d]$ into $M$ segments of integer length, times

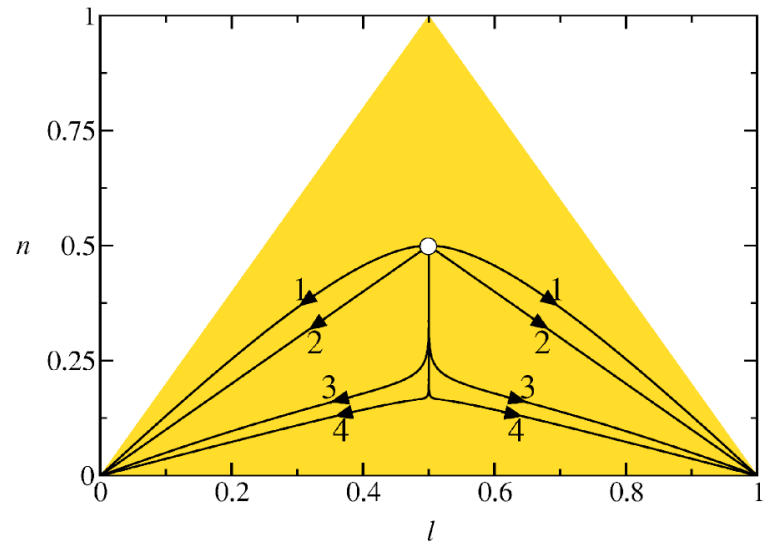

FIG. 2. Trajectories of the maxima of the exponent in Eq. (13) in the domain $\{l, n\}$, as temperature varies from $T=0$ (central point), to $T_{c}$ (lateral vertices, $b_{0}=0$ or $b_{0}=1$ ) at particular choices of parameters: (1) $p=0.01, I=1$; (2) $p=1 / 2, I=1$; (3) $p=0.1, I=0.1$; and (4) $p=1 / 2, I=0.1$. For each temperature, there are two equal contributions to free energy resulting from $b_{0}=1,0$.

the number of ways of dividing $[0,(N-L) / d]$ into $M+1$ integer segments (the last of them possibly zero):

$$
C_{M}^{(N-L) / d} C_{M-1}^{L / d-1}
$$

(where $C_{m}^{n}=n ! /[m !(n-m) !]$, a binomial coefficient).

Note, however, that our choice of $2 M$ coordinates $\left\{r_{1}, \ldots, r_{2 M}\right\}$ is constrained by the fact that the original problem has bonds connecting pairs of sites $q_{k}, q_{m}$. Therefore, if $q_{k} \in\left\{r_{1}, \ldots, r_{2 M}\right\}$ so must $q_{m}$. To take this fact into account, we multiply the previous factor by the normalizing factor,

$$
C_{M}^{p N} / C_{2 M}^{2 p N},
$$

which refers to the self-averaging property in the thermodynamic limit: That (almost) all possible pairings between the sites $\{d, 2 d, \ldots, N\}$ give the same $\Omega(L, M)$. The result for $\Omega(L, M)$ is, then:

$$
\Omega(L, M)=C_{M}^{2 p(N-L)} C_{M-1}^{2 p L-1} C_{M}^{p N} / C_{2 M}^{2 p N} .
$$

At this point, it is important to specify the variation range for the variables $L$ and $M$. Clearly, $0<M<p N$. On the other hand, since each $r_{i}-r_{i-1}$ measures at least $d=1 / 2 p$, we must have $2 p L \geqslant M$ and $p N-2 p L \geqslant M$, i.e., the before defined intensive variables $n=M / p N$ and $l=L / N$ should belong to the triangle

$$
n \leqslant 2 l, \quad n \leqslant 1-2 l, \quad 0 \leqslant n \leqslant 1,
$$

shown in Fig. 2.

For our purposes, all that will be required is the leading $\mathcal{O}(N)$ term in $\ln \Omega(L, M)$. Using Stirling's formula

$$
n ! \approx \sqrt{2 \pi n}\left(\frac{n}{e}\right)^{n},
$$

we arrive at

$$
\ln \Omega(L, M)=N s(l, n)
$$

with 


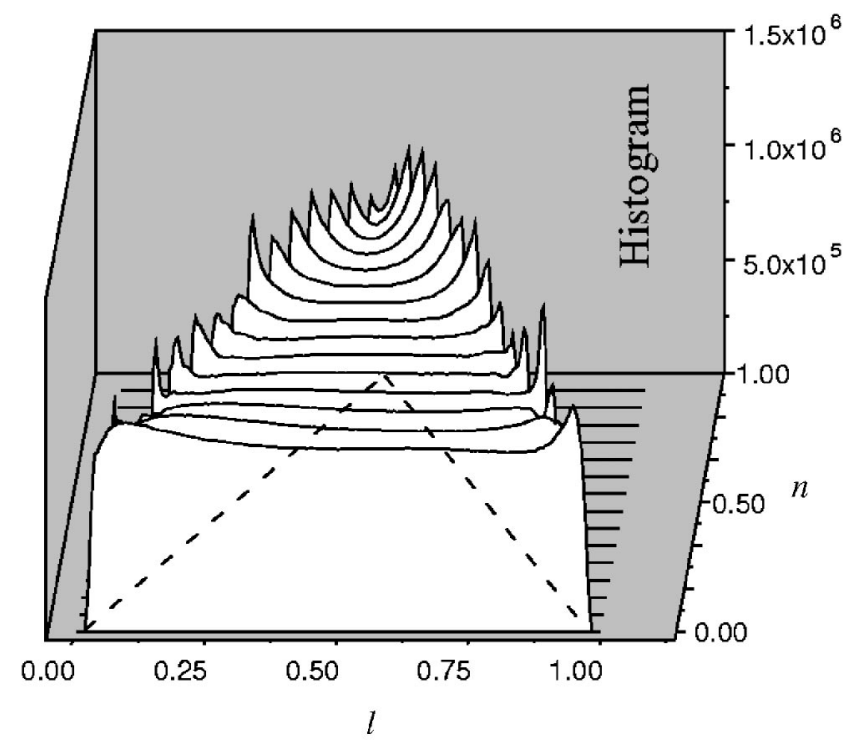

FIG. 3. Histogram of occurrences of $l$ values for several fixed values of $n$, obtained in multicanonical simulations with the entropy of Eq. (26) as sampling distribution for a sample with $p=1 / 4$ and $N=512$.

$$
\begin{aligned}
s(l, n)= & p[(2-2 l) \ln (2-2 l)+2 l \ln (2 l)+(1-n) \ln (1-n) \\
& -n \ln n-(2 l-n) \ln (2 l-n) \\
& -(2-2 l-n) \ln (2-2 l-n)]
\end{aligned}
$$

[dropping the terms $O(\ln N)$ ].

In order to confirm the basic assumption of selfaveraging, involved in the calculations leading to Eq. (26), we performed multicanonical Monte Carlo simulations on the variables $l$ and $n$. For the sampling probability distribution of a state $i$ (characterized by a set of values for the variables $\{b\})$, we used $p_{i}=1 / \Omega\left(L_{i}, M_{i}\right)$. The Monte Carlo move is given by:

(1) From an initial state $i$, propose a new state $j$, differing from $i$ by the value of a randomly chosen bond (with probability $1 / N_{b}$ ).

(2) Accept the new state with the usual probability $W_{i j}$ $=\min \left[1, \Omega\left(L_{i}, M_{i}\right) / \Omega\left(L_{j}, M_{j}\right)\right]$. This procedure ensures (by detailed balance and ergodicity of the algorithm) that the asymptotical sampling probability is $p_{i}$. The asymptotical histogram of frequencies in an $(l, n)$ point should, then, be given by

$$
H(l, n) \propto \Omega_{\alpha}(L, M) \frac{1}{\Omega(L, M)},
$$

where $\Omega_{\alpha}(L, M)$ is the actual number of states, with given $(L, M)$, of the sample that we are using in the simulation. If $\Omega_{\alpha}(L, M)=\Omega(L, M)$, the histogram should be flat in all the range of $(l, n)$.

The corresponding histograms (shown in Fig. 3, for a single disorder realization) are indeed quite flat, confirming that the entropies we calculated are apparently exact in the thermodynamic limit and very accurate for the moderate tested sizes. Recall that $\Omega(L, M) \propto \exp [N s(l, n)]$ and, for large $N$, even small deviations of $\ln \Omega_{\alpha}(L, M)$ from $S(l, n)$, would result in simulations which do not cover the entire spectrum, as ours do.

\section{THERMODYNAMIC BEHAVIOR}

The necessary conditions for the exponent in Eq. (13) to be maximum: $\partial f(l, n) / \partial n=\partial f(l, n) / \partial l=0$, define from Eq. (26) the following equations for the coordinates $n^{*}$ and $l^{*}$ of this maximum

$$
\begin{gathered}
\frac{n^{*}\left(1-n^{*}\right)}{\left(2-2 l^{*}-n^{*}\right)\left(2 l^{*}-n^{*}\right)}=t_{I}, \\
\frac{\left(2 l^{*}-n^{*}\right)\left(1-l^{*}\right)}{l^{*}\left(2-2 l^{*}-n^{*}\right)}=t_{J}^{d},
\end{gathered}
$$

$(d \equiv 1 / 2 p)$. These equations are easily solved:

$$
\begin{aligned}
& l^{*}=\frac{1-t_{J}^{d}-2 t_{I} t_{J}^{d}}{\left(1-t_{J}^{d}\right)^{2}-4 t_{I} t_{J}^{d}}, \\
& n^{*}=l^{*}\left(1+\frac{t_{I}-t_{J}^{d}}{1+t_{I}}\right),
\end{aligned}
$$

and, for $t_{I}, t_{J}$ varying with temperature, these are parametric equations for a certain trajectory of the maximum of the exponent in the $n, l$ plane [within the admissible range, Eq. (23)]. At zero temperature, when $t_{I}=t_{J}=1$, it locates at $l^{*}$ $=1 / 2, n^{*}=1 / 2$ (the central point in Fig. 2), corresponding to the maximum configurational entropy $s(l, n)$. As $T$ increases, the maximum moves toward the origin $n=l=0$ (or to $n=0$, $l=1$ ), as shown in Fig. 2. The trajectory reaches the very origin at a finite temperature $T=T_{c}$, given by the condition

$$
t_{J}^{d}\left(1+2 t_{I}\right)=1 \text {. }
$$

It is important to notice that below this critical temperature, when the reduced temperature $t \equiv T / T_{c}-1$ is nonzero $(t<0)$, no matter how small $|t|$ is, the coordinates of maximum $\left(l^{*}, n^{*}\right)$ Eqs. (28) and (29), are also finite: $l^{*}, n^{*} \sim|t|$. Hence, the corresponding numbers $L^{*}=l^{*} N$ and $M^{*}=p n^{*} N$ are macroscopic: $L^{*}, M^{*}=\mathcal{O}(N)$. On the other hand, the width of the maximum, estimated from the second derivatives $\partial^{2} s /\left.\partial l^{2}\right|_{l^{*}, n^{*}} \sim \partial^{2} s /\left.\partial n^{2}\right|_{l^{*}, n^{*}} \sim 1 / l^{*}$, is $\mathcal{O}\left(\sqrt{l^{*} / N}\right)$ and tends to zero in the thermodynamic limit, that is the probability distribution in macroscopic variables $l, n$ tends to a $\delta$-function with an amplitude which can only depend on macroscopic parameters of long-range links ( $p$ and $I$ ) but not on their specific realization. This justifies the above used assumption of self-averaging for the probability distribution and relates it to the known self-averaging property for observable values [18].

Above $T_{c}$, the maximum of the exponent in Eq. (13) goes away from the physical region, Eq. (23), while its highest value in this region is zero, attained at the origin. Hence, the last factor of the Eq. (13) turns $\mathcal{O}(1)$, and it gives no contribution to the free energy in the thermodynamic limit. Therefore, the free energy above $T_{c}$ is simply $f_{0}$, Eq. (14), but it 


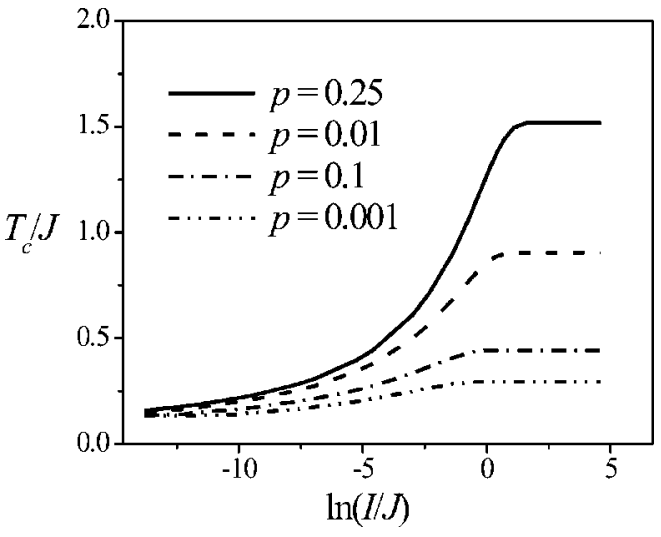

FIG. 4. Behavior of the critical temperature (in units of $J$ ) as a function of $I$ for several values of the concentration $p$ of shortcuts

gets an extra term below $T_{c}: f_{0}+f_{a}$, where $f_{a}$ is given by Eq. (15) with $l$ and $n$ are given by Eqs. (28) and (29). These equations combined provide a complete description of the thermodynamics of the model at all temperatures in zero field.

The dependence of transition temperature $T_{c}$, defined from Eq. (30), on the system parameters $p, J$, and $I$, can be easily analyzed in characteristic limits.

If shortcut bonds are much stronger than chain bonds, then for any finite $p$ and $I \rightarrow \infty$, the critical temperature tends to a finite value: $T_{c} \rightarrow J /\left(\operatorname{arctanh} 9^{-p}\right)$. Since shortcut spin pairs in this limit should be considered as single spins, the above value defines also the transition temperature for a random graph with connectivity 4 made of Ising chains of length $d=1 / 2 p$. In the limit of small concentration, $p \ll 1$, this critical temperature $T_{c}$ turns small compared to $J$, the energy scale for Ising chain:

$$
T_{c}=\frac{2 J}{\ln [1 /(p \ln 3)]},
$$

and such $I$-independent behavior holds as well for moderate shortcut strength (unless $I$ is too small: $I \ll J /|\ln (p \ln 3)|$ ). At last, in the limit where the shortcuts are much weaker than the chain bonds, $p I \ll J$, we have within logarithmic accuracy

$$
T_{c}=\frac{2 J}{\ln \left[T_{c} /(2 p I)\right]} \approx \frac{2 J}{\ln \{J /[p I \ln (J / p I)]\}} .
$$

The above relations define the system phase diagram in $p, I, T$ variables, as shown in Fig. 4.

It is of interest to compare these formulas to the finite critical temperatures, resulting from breaking down the Mermin-Wagner theorem for a two-dimensional Heisenberg magnet in the presence of (small) anisotropy $\Delta J \ll J: T_{c}$ $\approx J z / \ln \left(T_{c} / \Delta J\right)$ ( $z$ is the coordination number). They can also be referred to the percolation threshold $p_{c}$ for the onestate limit of the Potts model [19]: $T_{c}=2 J / \ln \left(1-p_{c}\right)$.

The nontrivial thermodynamics follows from the observation that close to the critical point, $0<-t \ll 1$, both variables $l$ and $n$ are $\mathcal{O}(|t|)$, while the trajectory $n(l)$ reaches the origin with asymptotic slope $d n / d l \rightarrow 4 /\left(1 / t_{I}+2\right)<4 / 3$, that is, always within the triangle, Eq. (23). Using Eqs. (28) and (29),

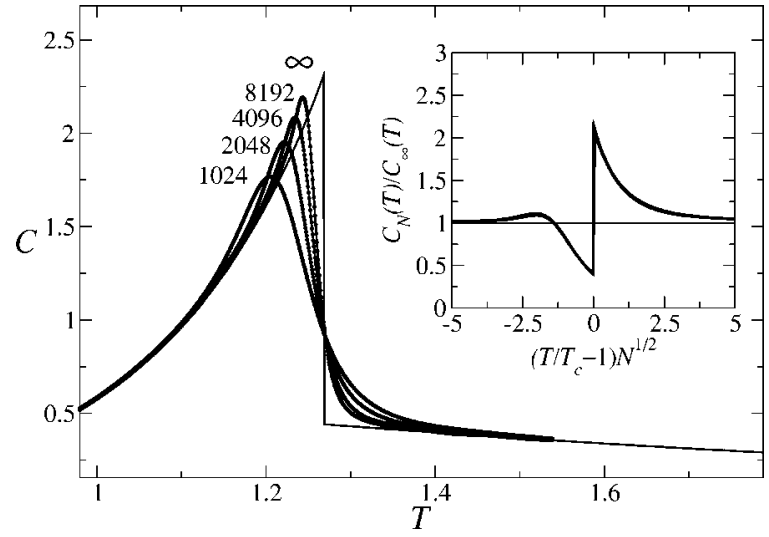

FIG. 5. Specific heat obtained by Monte Carlo simulations for four sizes of samples, with $p=1 / 4$ and $I=1$ in comparison with the theoretical curve $(\infty)$. Inset shows the collapse of the four curves when plotted in function of $t N^{1 / 2}$.

the additional term $f_{a}$ in the free energy can be simplified to:

$$
f_{a}=T p \ln \frac{\left(2-2 l^{*}-n^{*}\right)^{2}}{\left(1-n^{*}\right)\left(2-2 l^{*}\right)^{2}},
$$

and its leading terms in the critical region are clearly of order $\mathcal{O}\left(t^{2}\right)$. Hence, the specific heat per spin, $c_{v}=-T \partial^{2} f / \partial T^{2}$, has a finite jump $\Delta C$ at the critical point. As an example, we present in Fig. 5 the calculated exact specific heat for $p$ $=1 / 4$ and $I=J$, together with the results of simulations on samples of various sizes. This behavior permits one to classify the considered transition as second-order mean-fieldlike, with a critical exponent $\alpha=0$. In the case of $p \ll 1$ with $T_{c}$ given by Eq. (31), the jump is proportional to $p^{3} \ln ^{2}(p \ln 3)$, and is also independent of the shortcut bond strength $I$. When the shortcuts are much weaker than the chain bonds, $p I \ll J$, with $T_{c}$ by Eq. (32), we find the specific heat discontinuity proportional to $p(I / J)^{2} \ln ^{4}(J / p I)$. The above results indicate a nonanalytic decay of this mean-field amplitude at $p \rightarrow 0$, specific for the considered one-dimensional system. It is of interest to compare this to a power-law divergence in $p$ of the mean-field amplitudes for systems where a second-order transition at nonzero temperature exists in absence of longrange links [14].

\section{FINITE SIZE SCALING}

It was shown that the self-averaging property invoked for our calculation of $\Omega(L, M)$ is true in the thermodynamic limit, and flatness of the histograms in the multicanonical simulations suggests that the calculated $\Omega(L, M)$ is accurate (see Fig. 3). Nevertheless, there are visible deviations from flatness near the edges of the spectrum, which diminish with growing system size $N$. One can therefore ask whether our solution also contains the correct finite size scaling properties of this model.

To answer this question, the numerical sum of Eq. (12) was performed for different temperatures. The factorials were substituted by the Stirling's approximation and the specific heat was then obtained by numerical differentiation. The 


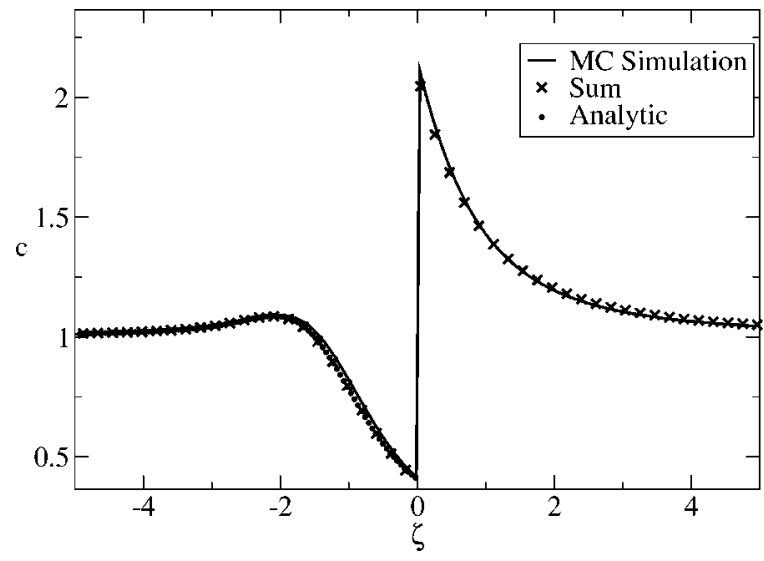

FIG. 6. Comparison between the Monte Carlo simulation for a sample of $N=8192$ and $p=1 / 4$, the numerical sum Eq. (12) and the analytical result (because of the small sizes, it was necessary to include corrections of $O\left(N^{-1 / 4}\right)$ to the finite size scaling).

comparison between the two independent calculations is presented in Fig. 6. Despite the fact that the Monte Carlo simulation was made for a unique sample (without disorder averaging), the agreement between these calculations is very good.

To derive the analytic form of the finite size scaling function, we approximated the sum in Eq. (12) by an integral in the intensive variables $l$ and $n$. Then, after the change of variables $(l, n) \rightarrow(l, u)$ where $u=n / l$, the partition function for a finite size $N$ of the sample becomes

$$
\mathcal{Z}_{N}=\exp \left[-\beta N\left(f_{0}+f_{a}\right)\right] \mathcal{Z}_{\mathrm{FSS}}
$$

where

$$
\mathcal{Z}_{\mathrm{FSS}}=\frac{N}{2 \pi p} \int_{0}^{+\infty} d l \int_{0}^{2} d u g(l, u) \exp (p N h(l, u))
$$

defines the contributions $\sim O\left(N^{-1} \ln N\right)$ into free energy $f$, with

$$
g(l, u)=\sqrt{\frac{2(1-l)}{(2-l(2+u))(2-u)}}
$$

and

$$
\begin{aligned}
h(l, u)= & 2(1-l) \ln \left(\frac{1-l}{1-l^{*}}\right)+(1-u l) \ln \left(\frac{1-u l}{1-u^{*} l^{*}}\right) \\
& -(2-u) l \ln \left(\frac{2-u}{2-u^{*}}\right)-u l \ln \left(\frac{u}{u^{*}}\right) \\
& -(2-l(2+u)) \ln \left(\frac{2-l(2+u)}{2-l^{*}\left(2+u^{*}\right)}\right) .
\end{aligned}
$$

The function $h(l, u)$ has a maximum at $\left(l^{*}, u^{*}\right)$. When $T$ $\rightarrow T_{c}^{-}$, we have $l^{*} \rightarrow 0^{+}$and $u^{*} \rightarrow 2 /\left[1+\operatorname{coth}\left(\beta_{c} I\right) / 2\right]<4 / 3$.

The integral in the Eq. (35) is dominated by the vicinity of the maximum. To obtain the leading order terms in $1 / N$ and $t$ we may expand $h(l, u)$ around the maximum,

$$
h(l, u) \approx-c_{1}\left(l-l^{*}\right)^{2}-c_{2} l\left(u-u^{*}\right)^{2},
$$

with definite constants $c_{1,2} \sim O(1)$, and replace $g(l, u)$ by $g\left(0, u^{*}\right)$. With a suitable change of variables, we obtain

$$
\mathcal{Z}_{\mathrm{FSS}}(\zeta) \approx \frac{N^{1 / 4}}{k_{1}} \int_{0}^{\infty} d x \int_{-y^{*}}^{\left(2 / u^{*}-1\right) y^{*}} d y e^{-\left(x-k_{2} \zeta\right)^{2}-x y^{2}},
$$

were $\zeta=t N^{1 / 2}, y^{*} \propto N^{1 / 4}$, and the constants $k_{1,2}$ are related to $c_{1,2}$. Since the finite size scaling limit is $N \rightarrow \infty$ and $t \rightarrow 0$ (at fixed $\zeta<0$ ), the limits of integration in $y$ tend to infinity and we get

$$
\mathcal{Z}_{\mathrm{FSS}}(\zeta) \approx \frac{\sqrt{\pi} N^{1 / 4}}{k_{1}} \int_{0}^{\infty} \frac{e^{-\left(x+k_{2} \zeta\right)^{2}} d x}{\sqrt{x}}
$$

This leads to a correction in the additional free energy:

$$
f_{a} \rightarrow f_{a}-T_{c} \frac{t^{2}}{\zeta^{2}} \ln \mathcal{Z}_{\mathrm{FSS}}(\zeta)
$$

and, since $f_{a}$ is also proportional to $t^{2}$, the scaling form for the specific heat becomes:

$$
\frac{C_{N}(T)}{C_{\infty}(T)}=c\left(t N^{1 / 2}\right)
$$

Thus the specific heat curves for finite size systems, $C_{N}(T)$, when scaled by $C_{\infty}(T)$ and plotted as a function of $t N^{1 / 2}$, should collapse to a single curve. The results of the Monte Carlo simulations are consistent with this prediction (see the inset of Fig. 5). An excellent accordance between the analytic behavior and the results of direct summation in Eq. (12) and of Monte Carlo simulations is shown in Fig. 6.

A similar scaling is observed in the susceptibility (not shown here) and has been observed by other authors in 1D [12] and also in 2D and 3D [13] (where $N=L^{d}$ is the number of spins, not the linear dimension $L$ of the lattice).

This is the expected form of scaling for a situation in which the dimensionality is greater than the upper critical dimension and hyperscaling is violated [20]. It is observed in all these small-world models for any dimension of the underlying regular lattice $[12,13]$. A similar steepest descent solution probably applies also in all of these cases.

\section{RELATION TO BETHE LATTICE APPROACH}

The local environment of a spin in our model looks like the Cayley tree in Fig. 7. The vertical links are shortcuts (of strength $I$ ) and the longer ones segments of the onedimensional chain, containing $d=1 / 2 p$ links of strength $J$. Since a shortcut from a given spin has an equal chance of linking it to anywhere in the lattice, we do not expect to find closed loops until we go $\mathcal{O}(\ln N)$ links away. Based on this insight, Dorogovtsev et al. [15] developed a description of the Ising model on such lattices based on the Bethe lattice solution. Their detailed results (namely for $T_{c}$ ) are not directly applicable to our lattices (which are not maximally random because of the strong correlation between shortcut 


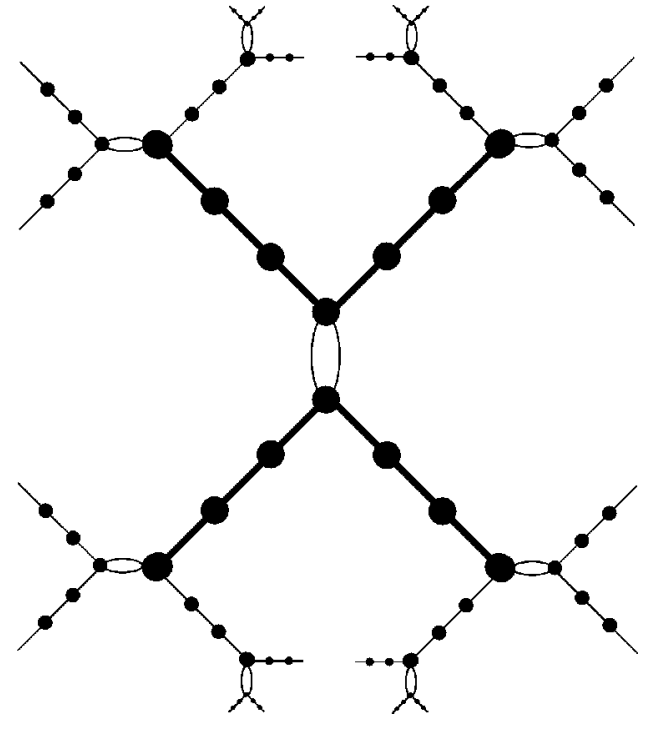

FIG. 7. The Bethe lattice that describes the local environment of any spin in the model: Shortcuts (double lines) between the sites divide the Ising chain into equal segments with $d$ (here, $d=3$ ) chain bonds (single lines).

sites). But their insight certainly is, and, since our results are not based on the Bethe lattice solution, and our lattice has a well defined thermodynamic limit, it is interesting to consider the relation between the two approaches.

In a Bethe lattice there is only one path to link two spins, as a result the correlation function is of a one-dimensional character. Therefore, the correlation between any two spins $\left\langle\sigma_{0} \sigma_{r}\right\rangle$ decays exponentially with the distance at any finite temperature (even at $T_{c}$ ). But the number of spins $\sigma_{r}$ at a given distance, $r$, from a given one, $N_{s}(r)$, grows exponentially with distance, not as a power law, as in a regular lattice. The function $\left\langle\sigma_{0} \sigma_{r}\right\rangle N_{s}(r)$ has a decay length that diverges at the ordering temperature of the Bethe lattice. It is straightforward to derive the exact transition temperature of a Bethe lattice of coordination $q, \beta_{c} J=\ln (q /(q-2)) / 2$, from this condition.

In our model, the chemical distance between two spins can be taken as $L^{\prime}+M$, where $M$ is the number of shortcuts and $L^{\prime} \geqslant M$ is the number of one-dimensional chain segments with $d$ bonds each, which connect these spins. Then, the one-dimensional correlator is

$$
\left\langle\sigma_{0} \sigma_{\left(L^{\prime}, M\right)}\right\rangle=e^{L^{\prime} d \ln t_{J}+M^{\prime} \ln t_{I}} .
$$

The total number of spins with this separation from a given one, is

$$
N_{s}\left(L^{\prime}, M\right)=2^{M} C_{M}^{L^{\prime}}
$$

therefore

$$
\left\langle\sigma_{0} \sigma_{\left(L^{\prime}, M\right)}\right\rangle N_{s}\left(L^{\prime}, M\right)=e^{-\kappa(x, T)\left(L^{\prime}+M\right)},
$$

where the decay constant $\kappa(x, T)$, with $x \equiv L^{\prime} /\left(L^{\prime}+M\right)$, is

$$
\begin{aligned}
\kappa(x, T)= & (1-x) \ln (1-x)+(2 x-1) \ln (2 x-1)-(1-x) \ln 2 \\
& -x \ln x-x \ln t_{J}^{d}-(1-x) \ln t_{I} .
\end{aligned}
$$

Minimizing $\kappa(x, T)$ with respect to $x$, we find that this minimum value $\kappa(T)=\min _{x} \kappa(x, T)$ decreases with temperature and turns zero just at $T=T_{c}$ given by Eq. (30). At any temperature above $T_{c}$ the function in Eq. (45) decays exponentially and there is no possibility of long-range order. In the language of the Bethe lattice, the occurrence of an extra term in the free energy below $T_{c}$, expresses the effect of boundaries, which is never negligible, no matter what the lattice size is, when the function in Eq. (45) does not decay with distance.

This analysis sheds some light on the previously stated violation of hyperscaling, which arises from the fact that the finite size corrections are not determined by the length that characterizes the decay of correlation functions. In fact, we have argued above that the spin-spin correlation function decays exponentially with the distance measured on the equivalent Bethe lattice, i.e., with the chemical distance on the original lattice. Thus, for the instance of $I=J$, we have $\xi=-1 / \ln t_{J}$ and

$$
\left\langle\sigma_{0} \sigma_{\left(L^{\prime}, M\right)}\right\rangle=e^{-\left(L^{\prime} d+M\right) / \xi} .
$$

It is well known that the chemical distance between any two randomly chosen spins is of order $\mathcal{O}(\ln N)$ so the correlation between two spins at a distance of order $N$ along the onedimensional chain is at least of order $N^{-1 / \xi}$; it does not decay exponentially with $N$ as the lattice and the distance between spins grow.

One interesting question that remains unanswered is whether one can modify the model in order to effectively be at or below the upper critical dimension, and therefore observe a non-mean-field behavior.

\section{CONCLUSIONS}

In summary, we have been able to derive an exact solution of an Ising model on a lattice with long range disordered interactions. This solution expresses the free energy in terms of the density of states as a function of two macroscopic variables of order $O(N)$, which therefore is self-averaging. Hence no disorder averaging is required in this approach. We obtained the thermodynamics in the $N \rightarrow \infty$ limit and also the finite size scaling behavior.

\section{ACKNOWLEDGMENTS}

The authors thank Sergei Dorogovtsev for pointing out the relation of our solution to the Bethe lattice. This work was financed by FCT (Portugal) and the European Union, through POCTI (QCA III). One of the authors (J.V.L.) was supported by FCT grant (Portugal) No. SFRH/BD/1261/2000 and another author (R.T.) by MCYT (Spain) and FEDER through project Nos. BFM2000-1108 and BFM2001-0341C02-01.

\section{APPENDIX}

The model in which the positions of the shortcut sites are randomly chosen can be solved along the same lines of the 
model considered in the main text. We denote the coordinates of the shortcut sites, in increasing order, by $\left\{q_{1}, q_{2}, \ldots, q_{2 p N}\right\}$ with $q_{1}>0$ (allowing for $q_{2 p N}=0$ ). The distances between consecutive sites are

$$
\begin{gathered}
d_{i}=q_{i}-q_{i-1}, \quad i \neq 1, \\
d_{1}=N-q_{2 p N}+q_{1},
\end{gathered}
$$

so that $\sum_{i=1}^{2 p N} d_{i}=N$. If we choose $M$ bonds (those for which $b=1$ ) from the total of $p N$, the corresponding shortcut sites $\left\{r_{1}, r_{2}, \ldots, r_{2 M}\right\} \subseteq\left\{q_{1}, q_{2}, \ldots, q_{2 p N}\right\}$ will divide the lattice into $2 M$ segments of lengths $l_{1}, \ldots, l_{2 M}$ where

$$
\begin{gathered}
l_{i}=r_{i}-r_{i-1}, \quad i \neq 1, \\
l_{1}=N-r_{2 M}+r_{1},
\end{gathered}
$$

and $\sum_{i=1}^{2 M} l_{i}=N$. Then, $\Omega(L, M)$ is the number of possible choices of the $M$ bonds such that

$$
l_{2}+l_{4}+l_{6}+\cdots+l_{2 M}=L .
$$

We define

$$
\Omega(L, M)=\Omega(M) P(L \mid M),
$$

where $\Omega(M)$ is the number of choices of $M$ shortcuts with $b=1$ from a total of $p N\left[\Omega(M)=C_{M}^{p N}\right]$, and $P(L \mid M)$ is the probability that any such choice of $M$ bonds will select $L$ chain bonds with $b=1$.

It should be stressed again that, for a given realization of disorder, this probability must be calculated in the event space consisting of the choices of $M$ shortcuts from the specific set of $p N$ random shortcuts. However, it follows from the self-averaging property in the thermodynamic limit that any statistically significant configuration of shortcuts leads to the same probability $P(L \mid M)$. In that case we can calculate it, enlarging the space of events to include all the configurations of shortcuts.

We are therefore led to ask in how many ways one can choose $2 M$ sites, $\left\{q_{1}, q_{2}, \ldots, q_{2 M}\right\} \subseteq\{1, \ldots, N-1\}$, such that the sum of even lengths in this series is $l_{2}+l_{4}+l_{6}+\cdots+l_{2 M}$ $=l$. We have seen above that it is given by

$$
C_{M}^{N-L} C_{M-1}^{L-1}
$$

Since the total number of such choices is $C_{2 M}^{N}$, we have

$$
P(L \mid M)=C_{M}^{N-L} C_{M-1}^{L-1} / C_{2 M}^{N},
$$

so that

$$
\Omega(L, M)=C_{M}^{p N} C_{M}^{N-L} C_{M-1}^{L-1} / C_{2 M}^{N} .
$$

With the same definitions as above, we get

$$
\begin{aligned}
s(l, n)= & -p n(\ln n / 4)-p(1-n) \ln (1-n) \\
& +(1-2 p n) \ln (1-2 p n)-(1-l-p n) \ln (1-l-p n) \\
& -(l-p n) \ln (l-p n)+(1-l) \ln (1-l)+l \ln l .
\end{aligned}
$$

The equation for the transition temperature is

$$
t_{J}\left(1+4 p t_{I}\right)=1
$$

with the characteristic limits

$$
\begin{gathered}
T_{c}=\frac{2 J}{\ln (1 / 2 p)}, \quad p \ll 1, \quad I>\frac{J}{\ln (1 / 2 p)}, \\
T_{c}=\frac{2 J}{\ln \left(T_{c} / 2 p I\right)}, \quad p I \ll J .
\end{gathered}
$$

The resulting thermodynamic behavior is essentially the same as in the model considered in the main text.
[1] G. A. Baker, Jr., Phys. Rev. 130, 1406 (1963).

[2] J. F. Nagle, Phys. Rev. A 2, 2124 (1970).

[3] D. J. Watts and S. H. Strogatz, Nature (London) 393, 440 (1998).

[4] D. J. Watts, Small Worlds (Princeton University Press, Princeton, NJ, 1999).

[5] R. Monasson, Eur. Phys. J. B 12, 555 (1999).

[6] L. F. L. Fernandez, R. Huerta, F. Corbacho, and J. A. Siguenza, Phys. Rev. Lett. 84, 2758 (2000).

[7] M. E. J. Newman and D. J. Watts, Phys. Rev. E 60, 7332 (1999).

[8] M. E. J. Newman, J. Stat. Phys. 101, 819 (2000).

[9] A. Goltsev, S. Dorogovtsev, and J. Mendes, Phys. Rev. E 67, 026123 (2003).

[10] A. Barrat and M. Weigt, Eur. Phys. J. B 13, 547 (2000).
[11] M. Gitterman, J. Phys. A 33, 8373 (2000).

[12] H. Hong, B. J. Kim, and M. Y. Choi, Phys. Rev. E 66, 018101 (2002)

[13] C. P. Herrero, Phys. Rev. E 65, 066110 (2002).

[14] M. B. Hastings, Phys. Rev. Lett. 91, 098701 (2003).

[15] S. Dorogovtsev, A. Goltsev, and J. Mendes, Phys. Rev. E 66, 016104 (2002).

[16] R. J. Baxter, Exactly Solved Models in Statistical Mechanics (Academic, New York, 1982).

[17] L. Saul and M. Kardar, Nucl. Phys. B 432, 641 (1994).

[18] I. Lifshitz, S. Gredeskul, and L. Pastur, Introduction to the Theory of Disordered Systems (Wiley, New York, 1982).

[19] P. Kasteleyn and C. Fortuin, J. Phys. Soc. Jpn. 26, 11 (1969).

[20] K. Binder, M. Nauenberg, V. Privman, and A. P. Young, Phys. Rev. B 31, 1498 (1985). 